\title{
Endoscopic endonasal transsphenoidal surgery using the iArmS operation support robot: initial experience in 43 patients
}

\author{
Toshihiro Ogiwara, MD, ${ }^{1}$ Tetsuya Goto, MD, ${ }^{1}$ Alhusain Nagm, MD, MSc, ${ }^{1,2}$ and Kazuhiro Hongo, MD \\ 1Department of Neurosurgery, Shinshu University School of Medicine, Matsumoto, Japan; and 2Department of Neurosurgery, \\ Al-Azhar University Faculty of Medicine-Nasr City, Cairo, Egypt
}

\begin{abstract}
OBJECTIVE The intelligent arm-support system, iArmS, which follows the surgeon's arm and automatically fixes it at an adequate position, was developed as an operation support robot. iArmS was designed to support the surgeon's forearm to prevent hand trembling and to alleviate fatigue during surgery with a microscope. In this study, the authors report on application of this robotic device to endoscopic endonasal transsphenoidal surgery (ETSS) and evaluate their initial experiences.
\end{abstract}

METHODS The study population consisted of 43 patients: 29 with pituitary adenoma, 3 with meningioma, 3 with Rathke's cleft cyst, 2 with craniopharyngioma, 2 with chordoma, and 4 with other conditions. All patients underwent surgery via the endonasal transsphenoidal approach using a rigid endoscope. During the nasal and sphenoid phases, iArmS was used to support the surgeon's nondominant arm, which held the endoscope. The details of the iArmS and clinical results were collected.

RESULTS iArmS followed the surgeon's arm movement automatically. It reduced the surgeon's fatigue and stabilized the surgeon's hand during ETSS. Shaking of the video image decreased due to the steadying of the surgeon's scopeholding hand with iArmS. There were no complications related to use of the device.

CONCLUSIONS The intelligent armrest, iArmS, seems to be safe and effective during ETSS. iArmS is helpful for improving the precision and safety not only for microscopic neurosurgery, but also for ETSS. Ongoing advances in robotics ensure the continued evolution of neurosurgery.

https://thejns.org/doi/abs/10.3171/2017.3.FOCUS16498

KEY WORDS endoscopic endonasal transsphenoidal surgery; robotic surgery; multimodality; iArmS; operation support system

$\mathrm{B}$ ECAUSE neurosurgery takes a relatively long time and requires delicate manipulations, hand trembling among neurosurgeons is a considerable obstacle to precise microneurosurgery. ${ }^{6}$ To resolve this problem, an operation support robot, an intelligent arm-support system called iArmS (DENSO), was developed with medical-engineering cooperation, including from Shinshu University. This is a revolutionary motorless medical robot that can support and maintain the ability of the surgeon's hand during microscopic neurosurgery. It freely follows the movement of the surgeon's arm without switch, and fixes and supports the arm of the practiced hand during manipulations. We have already reported on the innovativeness and usefulness of this automatic arm-support robot for microscopic neurosurgery. ${ }^{5,16}$
With the development of surgical instruments and improved surgical technique, endoscopic neurosurgery-in particular endoscopic endonasal transsphenoidal surgery (ETSS) for treatment of pituitary tumors and midline skull base lesions-has been expanded in neurosurgery. $1,3,4,9,10$ The application of the iArmS to ETSS has also been evaluated. This study presents in detail our initial experience with ETSS using iArmS in 43 patients, and we discuss the merits of this new device for ETSS.

\section{Methods \\ Patient Criteria}

We retrospectively analyzed data obtained in 43 patients ( 21 females and 22 males; mean age 51.2 years [range 


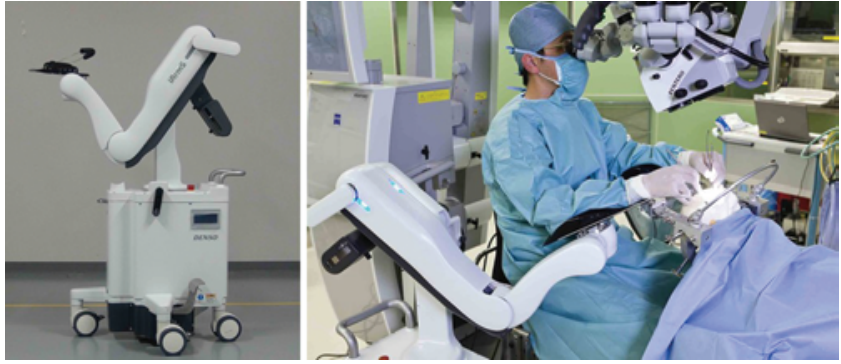

FIG. 1. Left: The iArmS weighs $97 \mathrm{~kg}$ and measures $600 \mathrm{~mm}$ in width, $600 \mathrm{~mm}$ in diameter, and 1100-1390 mm in height. Right: The arm holder of iArmS follows the surgeon's arm and automatically fixes it at an adequate position during surgery.

16-82 years]) who underwent iArmS-assisted ETSS at Shinshu University Hospital between April 2015 and September 2016. Cases involving a combined transcranialendonasal approach were excluded from this study. All patients underwent operations performed by the lead author (T.O.). The other Shinshu Neuroendoscopic Team member (A.N., the third author) coordinated the surgical paraendoscopic maneuvers (preoperative planning, devising a suitable layout, facilitating the multimodalities, assisting sellar reconstruction, and so on). The histological diagnoses included 29 pituitary adenomas, 3 meningiomas, 3 Rathke's cleft cysts, 2 craniopharyngiomas, 2 chordomas, 2 metastatic tumors, 1 angiosarcoma, and 1 adenoid cystic carcinoma. Among the 29 pituitary adenomas, 15 patients had hormone-secreting pituitary adenomas (9 with growth hormone-producing adenoma, 2 with Cushing's disease, 2 with prolactinoma, and 2 with thyroid-stimulating hormone-producing adenoma).

\section{iArmS: An Operation Support Robot}

The intelligent arm-support system, iArmS, was developed as an operation support robot. It was designed to support the surgeon's forearm to prevent hand trembling and to alleviate fatigue during microscopic neurosurgery (Fig. 1). It is used as an armrest for the surgeon. There are no motor systems in the joints, and it is controlled by force sensors, brakes, and encoders. The main unit of iArmS is available in 2 types: 1 for right-handed use and 1 for left-handed use. Surgeons can select 1 or both arms, as appropriate.

The arm holder of iArmS follows the surgeon's arm movement and automatically fixes it at an appropriate position using robotic technology. The system has 3 modes: transfer (Free), arm holding (Hold), and arm free (Wait). In the transfer mode, the arm holder follows the surgeon's arm passively. In the arm-holding mode, the arm holder is fixed and is used to support the weight of the surgeon's arm. The surgeon can change the position of the armrest while looking through the microscope and can continue the microsurgical procedure while holding surgical instruments (Video 1).

VIDEO 1. Clip demonstrating the system's 3 modes: transfer (Free), arm holding (Hold), and arm free (Wait). Each mode is converted automatically by analyzing signals from the force sensor and encoders in each joint. Copyright Toshihiro Ogiwara. Published with permission. Click here to view.

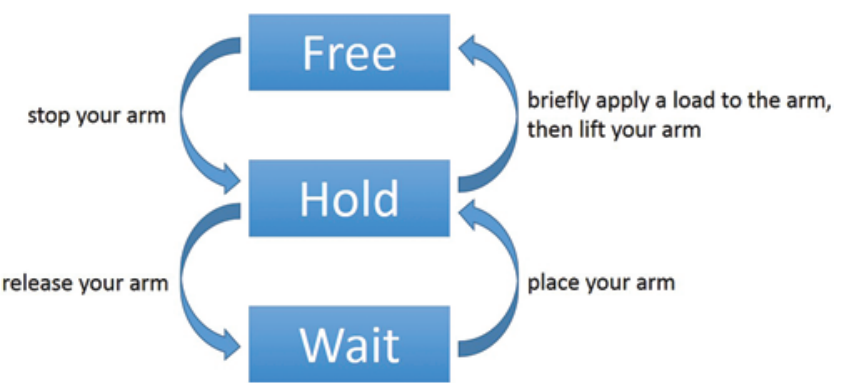

FIG. 2. A diagram of the robot's mechanical interfaces. The system has 3 modes: transfer (Free), arm holding (Hold), and arm free (Wait). When the surgeon's arm is placed on the arm holder, the mode changes from Wait to Hold. When the surgeon's arm moves to the desired position and holds still, the mode changes from Free to Hold. The mode changes from Hold to Free with a click action by the surgeon's arm.

Each mode is converted automatically by analyzing the signals from the force sensor and encoders in each joint (Fig. 2). This enables smooth, stress-free surgery.

Some special equipment is included in iArmS. For prevention of device malfunction, an alarm sounds when a load $>100 \mathrm{~N}$ is applied to the arm holder. When the surgeon's arm is suddenly removed from the arm holder for any reason, the arm is designed to stop automatically and the arm rises faster than the specified speed to avoid contact of the product with the patient. In addition, an emergency stop button is provided, and iArmS movement is strictly controlled with double power sensors. The importance of ensuring security is also emphasized.

\section{Clinical Application of iArmS}

Since September 2014, iArmS has been applied clinically in microscopic neurosurgery at Shinshu University Hospital (Fig. 3). Before surgery, the clinical application of iArmS was approved by the Ethics Committee of Shinshu University School of Medicine, and informed consent was obtained from all patients and their families. Since the device became commercially available in April 2015, it has been applied for ETSS.

\section{Operative Technique}

At our institution, a single surgeon from the Shinshu Neuroendoscopic Team performs ETSS with or without a scope holder, which is defined as the 2-hand technique; the 4-hand technique with a scope-holding assistant is not applied. ${ }^{2}$ A navigation system is routinely used for intraoperative confirmation of anatomical location. A rigid scope $\left(4 \mathrm{~mm}\right.$ in diameter, $18 \mathrm{~cm}$ in length, with angled lenses $\left[0^{\circ}\right.$ and $30^{\circ}$; ; Karl Storz) is used.

While under general anesthesia with orotracheal intubation, the patient is placed supine on the table, and the head is fixed in a Sugita head holder. The neck is slightly extended, and the head is gently turned to the right toward the surgeon to permit a good view via the nostrils. After standard preparation for ETSS procedures, iArmS (covered with a sterile sheet) is introduced into the operative field. The endonasal transsphenoidal approach is performed through the nostrils bilaterally via the right paraseptal approach with left rescue flap following decongestion of the nasal mucosa with epinephrine gauze. ${ }^{13}$ 


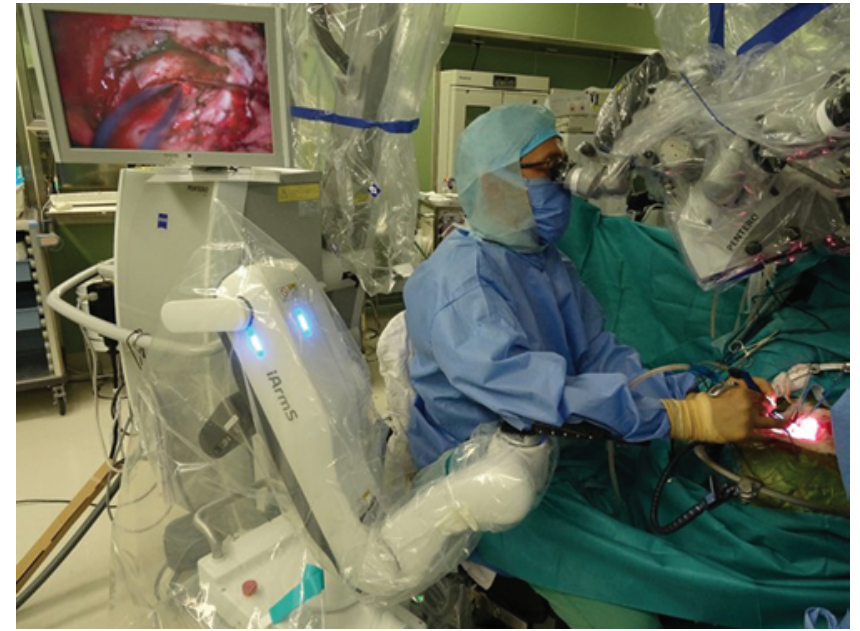

FIG. 3. Photograph showing microscopic neurosurgery with iArmS. The arm holder follows the surgeon's arm and automatically fixes it at an appropriate position and supports the surgeon's arm.

The anterior wall of the sphenoid sinus and the intersinus septum are resected using Kerrison punches and a high-speed drill for wide sphenoidotomy. During this nasal-sphenoid phase of the procedure, the surgeon holds the endoscope in the nondominant hand supported with iArmS and the surgical instrument in the free dominant hand (Fig. 4). After the anterior sphenoidectomy is completed, the role of iArmS is finished. A UniArm endoscope holder (Mitaka Kohki) is introduced and the endoscope is fixed to it. ${ }^{1}$ This provides the surgeon with a steady video image and frees both hands to maneuver the surgical instruments.

\section{Clinical Evaluation}

This study reviews a single surgeon's initial experience using the iArmS; the evaluation of effectiveness of its robotic device for ETSS was based only on the surgeon's subjectivity. This is a preliminary report of an initial experience, and iArmS is applied only during the nasal and sinus phases. Surgical outcomes (including tumor removal rate, complication rate, operative time, and blood loss) are mainly attributed to the sellar and reconstruction phases in ETSS for skull base lesions. From this point of view, the fatigue reduction of the surgeon's arm may indirectly lead to improvement of the surgical outcome. However, it seems to be difficult to prove the direct effectiveness of iArmS for ETSS at this initial stage. The improved surgical outcome will be demonstrated at the next stage.

\section{Results}

Application of the iArmS in the current series was easy (despite its steep learning curve) because we had sufficient knowledge of its characteristics and were familiar with its manipulation before performing surgery. The surgeon's arm moved freely (that is, without resistance or heaviness) while using iArmS. There was no need to push any buttons to convert between locked and free modes, thus solving the problems associated with freely movable armrests, such as the need for time-consuming and complex
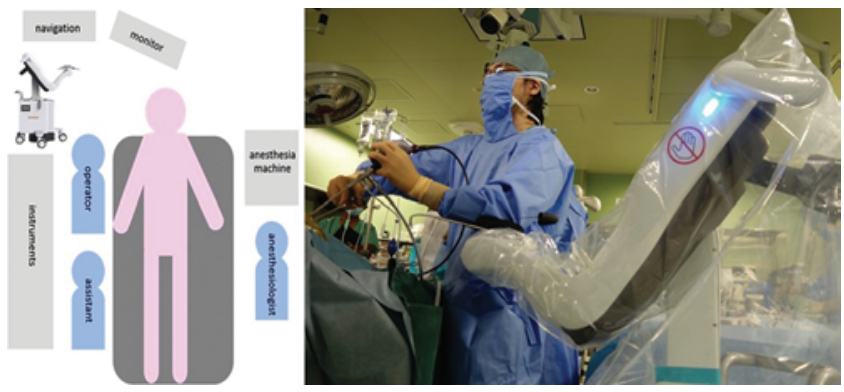

FIG. 4. Layout of the operating theater (left) and a photograph (right) showing endoscopic ETSS during the nasal phase with iArmS. The system supports the surgeon's nondominant hand, which holds the endoscope.

adjustments. The iArmS system decreased the surgeon's fatigue, reduced hand trembling, and allowed the surgeon to remain comfortable throughout the procedure. Shaking in the video image decreased due to the steadying of the surgeon's scope-holding hand with iArmS (Video 2).

VIDEO 2. Clip showing a comparison between the procedures with and without iArmS. The hand that is supported by iArmS is more stable than the free hand during the nasal phase. Copyright

Toshihiro Ogiwara. Published with permission. Click here to view.

The surgeon's arm did not compress the arm holder more than the allowable load resistance of iArmS (100 N) during the procedures, although overload causes emergency cessation of the system. There were no complications related to the use of this system in any of the cases.

\section{Discussion}

\section{Endoscopic Endonasal Surgery and Multimodality}

ETSS is a minimally invasive surgery suitable for brain tumors, such as pituitary adenomas and meningiomas. With the development of surgical techniques and improvement of instruments, indications for ETSS have expanded, and it has shown rapid advancement over the past several decades. ${ }^{1,3,4,9,10}$ However, surgical difficulties and risks of ETSS are increasing, and there are some practical limitations with respect to this procedure. Consequently, a safe operation requires the surgeon to be highly skilled, and the development of new instruments and modalities are required. Some additional modalities (such as neuronavigation, 3D endoscopy, and indocyanine green videoangiography) are necessary in the armamentarium of the neurosurgeon performing ETSS to enhance the surgical results and to prevent surgical complications. . $^{7,8,11,14,15}$

Robotic technology could also become an essential modality for ETSS. Such technology would allow a comfortable room in the operating theater and facilitate stable and accurate surgery based on the delicate manipulations required, even in such a deep and narrow operative field. The ETSS technique can be simplified and refined using robotic technology as well as by modification of surgical instruments. ${ }^{9}$ One possible application of robotic technology for ETSS is in support of the routine technique. We noticed the importance of stabilizing the surgeon's arm during the procedure and have developed an intelligent arm-support system, EXPERT, which was the prototype of iArmS. ${ }^{5,6,16}$ 


\section{ETSS With iArmS}

Neurosurgeons generally place their finger and/or hypothenar eminence beside the operating field to reduce hand trembling in microscopic neurosurgery. Some armrests (such as rigid bar, wrist support, and hand rest) have also been developed for microsurgery. ${ }^{6}$ However, these devices are not suitable to making a large movement with the arm such as is required in endoscopic surgery, and there are few places to put the hand during ETSS.

Stabilization of the surgeon's hand and alleviation of fatigue can facilitate stable and accurate surgery. In addition, a stable video image enables detailed inspection of several structures in the operative field. Use of a scope holder that fixes the endoscope also prevents shaking of video images. However, when using a scope holder, the surgeon must stop the surgical procedure each time the endoscope is moved to a suitable position. Furthermore, the spatial orientation is difficult to understand in the operative field without a continuous freely moving scope. Endoscopic neurosurgeons observe the depth of the operative field via 2D video images using dynamic stereo vision by allowing the scope to move back and forth slightly.

Our 2-hand technique provides perfect command of not only the instrument but also the endoscope, and iArmS provides both stability of the surgeon's hand and excellent operability during ETSS. During the nasal and sphenoid phases, in which simultaneous use of 2 instruments is not essential, our 2-hand technique with iArmS is superior to that with a scope holder from the viewpoint of understanding the tertiary structure, reducing operation time, and achieving excellent operability. During the sellar and reconstruction phases, simultaneous use of 2 instruments controlled by both hands is applied in the 2-hand technique with a scope holder.

However, the 4-hand technique, in which an assistant holds the scope with iArmS, may be better than our method. This technique enables delicate scope movement in collaboration with an operator without losing stability of the video image. Applying this 4-hand technique may allow the use of iArmS during the whole ETSS from the nasal to the reconstruction phases. Thus, iArmS is a novel intelligent armrest using robotic technology that is suitable not only to microscopic neurosurgery but also to endoscopic neurosurgery.

\section{Limitations of the Study}

There were some limitations in this study. The most significant issue was that the usefulness of iArmS could not be confirmed statistically, because we did not examine whether it could reduce fatigue during ETSS. However, it is reasonable to speculate that a well-designed armrest would reduce such fatigue, as shown in Video 2. Furthermore, it is not easy to make a scientific assessment of a neurosurgeon's fatigue, but further studies of this issue are warranted.

One disadvantage is that the space occupied by iArmS is a problem. This is because many surgical tools (such as the operative endoscopic system, navigation systems, drilling systems, and electrocoagulation system) must be set near the surgeon. The base of iArmS is large and heavy to prevent the system from falling over. Consideration of

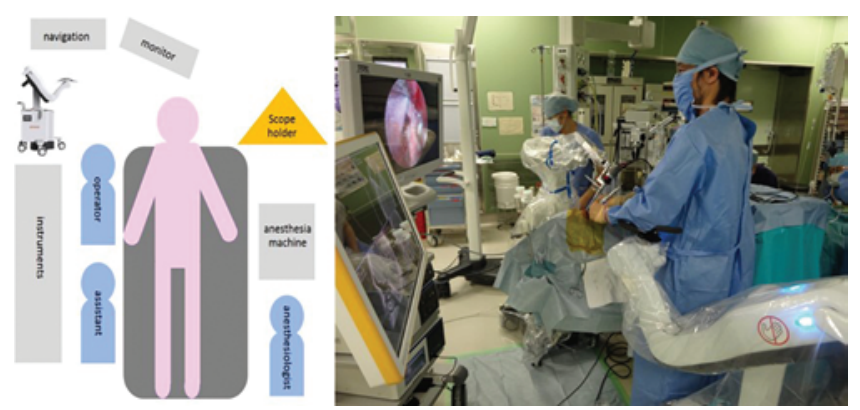

FIG. 5. Layout of the operating room (left) and a photograph (right) showing endoscopic ETSS during the sellar phase with iArmS. The system supports the surgeon's nondominant hand, which holds various surgical instruments. The endoscope is held with a scope holder on the opposite side of the surgeon.

the layout of the busy operating room is necessary. Due to this issue, the use of iArmS during sellar and reconstruction phases, in which manipulation requires 2 hands, has not been satisfactorily evaluated.

However, we maximized the possible usefulness of this system by devising a suitable layout around the operation table (Fig. 5). Reducing the size of the iArmS while maintaining its functionality will be necessary in future development of the system. The greatest drawback to existing surgical robots is cost performance. The iArmS is currently priced at approximately 98,000 US dollars, which is too expensive for most institutions; this will inhibit the worldwide adoption of iArmS. However, it would be worthwhile to examine its price and running costs.

Next-generation robotic platforms may mitigate these limitations. Over time, the large and expensive multipurpose robots of today are likely to be replaced by smaller and more affordable robots that are tailored to particular procedures. ${ }^{12}$ Further improvements in robotic technology and the accumulation of clinical data are needed to evaluate the usefulness of iArmS for ETSS.

\section{Conclusions}

We have reported our initial experience with iArmS in ETSS. iArmS, which is considered a breakthrough operation support robot, enables stability of the surgeon's hand and excellent operability in ETSS. Sufficient knowledge of the characteristics of iArmS makes it a useful new modality for ETSS. It is expected that it will lead to the development and widespread adoption of robotic neurosurgery. Although robotic platforms have the potential to greatly enhance the performance of ETSS, there is a strong rationale for research into next-generation robots that are better suited to ETSS. Ongoing advances in surgical technique and instruments as well as robotics will ensure brisk evolution in this expanding field.

\section{References}

1. Cappabianca P, Cavallo LM, Solari D, Stagno V, Esposito F, de Angelis M: Endoscopic endonasal surgery for pituitary adenomas. World Neurosurg 82 (6 Suppl):S3-S11, 2014

2. Castelnuovo P, Pistochini A, Locatelli D: Different surgical approaches to the sellar region: focusing on the "two nostrils four hands technique." Rhinology 44:2-7, 2006 
3. Cavallo LM, Solari D, Esposito F, Cappabianca P: Endoscopic endonasal approach for pituitary adenomas. Acta Neurochir (Wien) 154:2251-2256, 2012

4. Gondim JA, Almeida JP, Albuquerque LA, Gomes EF, Schops M: Giant pituitary adenomas: surgical outcomes of 50 cases operated on by the endonasal endoscopic approach. World Neurosurg 82:e281-e290, 2014

5. Goto T, Hongo K, Yako T, Hara Y, Okamoto J, Toyoda K, et al: The concept and feasibility of EXPERT: intelligent armrest using robotics technology. Neurosurgery 72 (Suppl 1):39-42, 2013

6. Hara Y, Goto T, Okamoto J, Okuda H, Iseki H, Hongo K: An armrest is effective for reducing hand tremble in neurosurgeons. Neurol Med Chir (Tokyo) 55:311-316, 2015

7. Hide T, Yano S, Shinojima N, Kuratsu J: Usefulness of the indocyanine green fluorescence endoscope in endonasal transsphenoidal surgery. J Neurosurg 122:1185-1192, 2015

8. Inoue A, Ohnishi T, Kohno S, Nishida N, Nakamura Y, Ohtsuka Y, et al: Usefulness of an image fusion model using three-dimensional CT and MRI with indocyanine green fluorescence endoscopy as a multimodal assistant system in endoscopic transsphenoidal surgery. Int J Endocrinol 2015:694273, 2015

9. Jho HD, Carrau RL: Endoscopic endonasal transsphenoidal surgery: experience with 50 patients. J Neurosurg 87:44-51, 1997

10. Kassam AB, Prevedello DM, Carrau RL, Snyderman CH, Thomas A, Gardner P, et al: Endoscopic endonasal skull base surgery: analysis of complications in the authors' initial 800 patients. J Neurosurg 114:1544-1568, 2011

11. Litvack ZN, Zada G, Laws ER Jr: Indocyanine green fluorescence endoscopy for visual differentiation of pituitary tumor from surrounding structures. J Neurosurg 116:935-941, 2012

12. Marcus HJ, Hughes-Hallett A, Cundy TP, Yang GZ, Darzi A, Nandi D: da Vinci robot-assisted keyhole neurosurgery: a cadaver study on feasibility and safety. Neurosurg Rev 38:367-371, 2015

13. Otto BA, Bowe SN, Carrau RL, Prevedello DM, Ditzel Filho
LF, de Lara D: Transsphenoidal approach with nasoseptal flap pedicle transposition: modified rescue flap technique. Laryngoscope 123:2976-2979, 2013

14. Pennacchietti V, Garzaro M, Grottoli S, Pacca P, Garbossa $\mathrm{D}$, Ducati A, et al: Three-dimensional endoscopic endonasal approach and outcomes in sellar lesions: a single-center experience of 104 cases. World Neurosurg 89:121-125, 2016

15. Thirumala PD, Kodavatiganti HS, Habeych M, Wichman K, Chang YF, Gardner P, et al: Value of multimodality monitoring using brainstem auditory evoked potentials and somatosensory evoked potentials in endoscopic endonasal surgery. Neurol Res 35:622-630, 2013

16. Yako T, Goto T, Hongo K: Usefulness and limitation of a freely movable armrest in microneurosurgery. Int $\mathbf{J}$ Neurol Neurosurg 1:185-190, 2009

\section{Disclosures}

The authors report no conflict of interest concerning the materials or methods used in this study or the findings specified in this paper.

\section{Author Contributions}

Conception and design: Goto, Ogiwara. Acquisition of data: Ogiwara, Nagm. Analysis and interpretation of data: Ogiwara, Nagm. Critically revising the article: Goto, Hongo. Reviewed submitted version of manuscript: all authors. Study supervision: Goto, Ogiwara.

\section{Supplemental Information \\ Videos}

Video 1. https://vimeo.com/207450348.

Video 2 . https://vimeo.com/207450441.

\section{Correspondence}

Tetsuya Goto, Department of Neurosurgery, Shinshu University School of Medicine, 3-1-1 Asahi, Matsumoto 390-8621, Japan. email: tegotou@shinshu-u.ac.jp. 